Muséologies

Les cahiers d'études supérieures

muséologies

\title{
Les nouveaux rôles des musées et la diversité culturelle : l'exemple de Tate Encounters, Britishness and Visual Culture, Tate Britain
}

\section{Sophie Orlando}

Volume 6, numéro 1, 2012

URI : https://id.erudit.org/iderudit/1011530ar

DOI : https://doi.org/10.7202/1011530ar

Aller au sommaire du numéro

Éditeur(s)

Association Québécoise de Promotion des Recherches Étudiantes en Muséologie (AQPREM)

ISSN

1718-5181 (imprimé)

1929-7815 (numérique)

Découvrir la revue

Citer cet article

Orlando, S. (2012). Les nouveaux rôles des musées et la diversité culturelle : l'exemple de Tate Encounters, Britishness and Visual Culture, Tate Britain. Muséologies, 6(1), 15-33. https://doi.org/10.7202/1011530ar

\section{Résumé de l'article}

Pour faire écho à la nouvelle image de la Grande-Bretagne, multiculturaliste, plurielle et inclusive, le gouvernement de Tony Blair met en place, de 1997 à 2010, une politique de "diversité culturelle ». L’omniprésence de celle-ci dans les débats nationaux va désormais lier définitivement les politiques culturelles du gouvernement à celle des musées nationaux britanniques. Dans cet article, Sophie Orlando prend comme cas d'étude les politiques curatoriales et éducatives du Tate Britain, et plus particulièrement le programme de recherche " Tate Encounters ", qui tente de relire les politiques de diversité culturelle et d'intégration sociale, les différentes conceptions de la diaspora et de l'identité nationale, tout en interrogeant le rôle de l'institution muséale dans ces débats. L'auteure analyse ainsi les conditions d'émergence du projet et observe sa progression théorique, abordant les enjeux du programme de recherche et les problématiques qui ont jalonné l'expérience, de 2006 à 2011. Sophie Orlando commente finalement certains des résultats obtenus, notamment méthodologiques, et se questionne sur l'analyse du programme quant aux relations entre politique et culture qui entrent en jeu dans la promotion de la diversité culturelle du Tate Britain.
Tous droits réservés (C) Association Québécoise de Promotion des Recherches Étudiantes en Muséologie (AQPREM), 2012
Ce document est protégé par la loi sur le droit d'auteur. L’utilisation des services d'Érudit (y compris la reproduction) est assujettie à sa politique d'utilisation que vous pouvez consulter en ligne.

https://apropos.erudit.org/fr/usagers/politique-dutilisation/ 
Article un

Les nouveaux rôles des musées

et la diversité culturelle: l'exemple

de Tate Encounters, Britishness

and Visual Culture, Tate Britain

Sophie Orlando 
Pour faire écho à la nouvelle image de la Grande-Bretagne, multiculturaliste, plurielle et inclusive, le gouvernement de Tony Blair met en place, de 1997 à 2010, une politique de "diversité culturelle». L'omniprésence de celle-ci dans les débats nationaux va désormais lier définitivement les politiques culturelles du gouvernement à celle des musées nationaux britanniques. Dans cet article, Sophie Orlando prend comme cas d'étude les politiques curatoriales et éducatives du Tate Britain, et plus particulièrement le programme de recherche "Tate Encounters", qui tente de relire les politiques de diversité culturelle et d'intégration sociale, les différentes conceptions de la diaspora et de l'identité nationale, tout en interrogeant le rôle de l'institution muséale dans ces débats. L'auteure analyse ainsi les conditions d'émergence du projet et observe sa progression théorique, abordant les enjeux du programme de recherche et les problématiques qui ont jalonné l'expérience, de 2006 à 2011 . Sophie Orlando commente finalement certains des résultats obtenus, notamment méthodologiques, et se questionne sur l'analyse du programme quant aux relations entre politique et culture qui entrent en jeu dans la promotion de la diversité culturelle du Tate Britain.

Sophie Orlando est historienne de l'art, docteure en art contemporain diplômée de l'Université de Paris 1 Panthéon-Sorbonne. Elle occupe un poste d'enseignement en tant qu'ATER à I'Université Paul-Valéry de Montpellier en France. Spécialiste des politiques identitaires dans la Grande-Bretagne des années 1980 à nos jours, elle s'intéresse aux relations entre les politiques culturelles et les stratégies artistiques, à la manière dont les représentations identitaires sont utilisées par les différents acteurs de l'art contemporain (institutions, artistes, historiens). Elle est directrice de l'association One Piece at a Time, groupe d'études interdisciplinaires sur les arts britanniques, et corédactrice de la revue en ligne Pied-à-terre. Elle développe actuellement une recherche sur la mondialisation artistique à l'époque contemporaine à travers les disciplines issues des sciences humaines, et en particulier les études postcoloniales, les études de genres, les études culturelles et la muséologie. Elle coédite avec Catherine Grenier une Anthologies de textes fondamentaux sur l'art et la mondialisation depuis 1950 à nos jours, à paraître aux éditions du Centre Pompidou à l'automne 2012. Université Paul-Valéry, Montpellier so.orlando@gmail.com 
L'interprétation des identités collectives par les mondes de l'art britannique s'est principalement nourrie de trois éléments dans les 50 dernières années: la théorisation de l'histoire postcoloniale par Rasheed Araeen, Homi K. Bhabha, Stuart Hall, Paul Gilroy, Gayatri C. Spivak, ou Edward W. Said ${ }^{1}$, la volonté de reconnaissance des Black Artists et l'omniprésence des débats d'abord sur l'ethnicité, puis sur la "diversité culturelle». Ce terme s'impose lors de l'instauration de la Cool Britannia, un programme de revalorisation de l'identité culturelle britannique établi par Tony Blair et qui vise à penser les conséquences culturelles de la décolonisation et à comprendre le développement de nouvelles identités sur un même territoire. Le premier ministre souhaite interroger les valeurs des identités plurielles de la Grande-Bretagne au regard des disparités régionales et des revendications (le particularisme gallois, la dominance britannique sur l'Écosse, la scission violente des troubles irlandais) et des vagues d'immigration du Commonwealth. Il ne s'agit pas de construire un multiculturalisme homogène, mais des relations interethniques. L'institutionnalisation de la notion de diversité culturelle s'est surtout forgée au sein de l'Arts Council et du ministère de la Culture, des Médias et du Sport (DCMS) à partir des années 1990. Au même moment, David Anderson, secrétaire d'État au Service du patrimoine et directeur du Département interprétation et apprentissage du Victoria and Albert Museum (V\&A), est invité à rédiger un rapport sur le rôle de l'éducation dans les musées. Il soumet son étude A Common Wealth, Museum in the Learning Age en 1997 au gouvernement de Tony Blair, dans laquelle il explique que les musées du XXI siècle devront devenir des centres d'apprentissage dont les expositions, les programmes d'événements et les autres outils seront mis au profit de l'autonomie et de la participation du spectateur. Le rôle du musée est pointé du doigt ; il est désigné comme un lieu d'expérimentation, de

1 ARAEEN, Rasheed. Making Myself Visible. Londres: Kala Press, 1984 ; BHABHA, Homi K., The Location of Culture. New York, Londres : Routledge, 2004; STUART, Hall. «Nouvelles ethnicités». Traduit dans HALL, Stuart. Identités et cultures, politiques des cultural studies. Paris: Amsterdam, 2008; GILROY, Paul. L'Atlantique noir, promotion, de création des identités collectives. Le musée doit désormais s'adresser à un spectateur émancipé, capable d'accéder librement aux œuvres d'art, de se reconnaître dans les récits qu'elles soutiennent et d'y puiser les grands traits d'une culture diversifiée de l'histoire contemporaine du pays. Vont se développer conjointement une stratégie éducative et une stratégie marketing des publics, une réévaluation du rôle du musée dans la société et des techniques d'accroissement du nombre de visiteurs, une conception centrée sur les particularismes identitaires, économiques et culturels du spectateur et sa nécessaire identification à une histoire collective. L'omniprésence de la question des débats nationaux sur la diversité culturelle va désormais lier définitivement les politiques culturelles du gouvernement à celle des musées nationaux britannique. Comment le Tate Britain interprète-t-il la diversité culturelle? La représentation de la diversité dans les institutions muséales semble s'être progressivement alignée dans le sillon du programme blairiste d'intégration sociale et de représentation des identités britanniques plurielles. Après une introduction portant sur les enjeux de la diversité culturelle, cet article prendra pour cas d'étude les politiques curatoriales et éducatives du Tate Britain, mais surtout le programme de recherche intitulé Tate Encounters: Britishness and Visual Culture.

\section{La diversité culturelle}

Dans le catalogue Les magiciens de la terre, Homi K. Bhabha définissait ainsi la diversité culturelle:

La diversité culturelle est un objet épistémologique (la culture comme objet de connaissance empirique), tandis que la différence culturelle est un phénomène de sens, par quoi les énoncés de la culture et sur la culture opèrent des distinctions et autorisent la production de domaines de référence,

Modernité et double conscience. Paris: Amsterdam [1993] 2009; SPIVAK, Gayatri Chakravorty. Les Subalternes peuvent-elles parler? Paris: Amsterdam [1988] 2009. 2 Publié en 1999: ANDERSON, David. A Common Wealth, Museums in the Learning Age. Londres: DCMS, 1999. 
d'application et de compétence. La diversité culturelle est la reconnaissance de " contenus » et usages culturels donnés d'avance, maintenus dans le cadre culturel du relativisme. Elle donne naissance à des notions rassurantes de multiculturalisme, d'échanges culturels, ou de culture de l'humanité. La diversité culturelle est aussi la représentation d'une rhétorique de la séparation de cultures unifiées qui n'ont pas été souillées par l'intertextualité de leur situation historique, et survivent dans l'utopie du souvenir mythique d'une identité unique ${ }^{3}$.

Ainsi la diversité culturelle prétend dépasser les différences de classes, de genres, d'origines et de religions. Bhabha souligne le mécanisme du principe de diversité culturelle en tant que mode de connaissance et d'appréhension du monde universaliste en tant que caractéristique fondamentale de l'humanité. Les institutions culturelles jouent avec cette négociation, ce glissement entre la désignation de la diversité et son utilisation pour définir des identités spécifiques et, par extension, des types de publics, de cultures, voire des types d'arts (art contemporain africain, art contemporain asiatique, etc.). Dans l'usage de la notion de diversité culturelle est également suggéré l'individu, son humanisme, sa liberté, son égalité. Le multiculturalisme, selon Bhabha, est une administration de la relation entre l'individu et le pluralisme, là où la politique de la différence construit l'altérité. Celui-ci préfère construire une étude de l'hybridité, du métissage qui naît de la traduction culturelle des différences. L'hybridité rend possible le "tiers-espace", un lieu d'annihilation des binarismes. Bhabha n'a pas tort lorsqu'il pointe une ambiguïté au sein du rapport de l'Occident à la diversité culturelle. À l'instar de Paul Gilroy, caractérisant la double conscience du Noir américain

3 BHABHA, Homi K. Les magiciens de la terre. Paris: Centre Pompidou, p. 25.

4 GILROY, Paul. L'Atlantique noir, Modernité et double

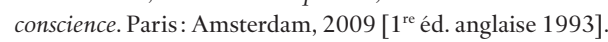

5 KHAN, Naseem. The Arts Britain Ignores: The Arts of Ethnic Minorities in Britain. Grande-Bretagne: Community Relations Commission, 1976.

6 DCMS, ou Department of Culture, Medias and Sport. vis-à-vis de sa posture à la fois subalterne et dominante ${ }^{4}$, s'inscrit au cœur de la diversité culturelle la projection du désir d'être à la fois dans la reconnaissance de l'Autre et dans la création de la division entre soi et l'Autre, un droit à l'égalité dans la différence et, en même temps, le principe humaniste et universaliste, propre au mécanisme de pensée de l'Europe moderne.

En Grande-Bretagne, la diversité culturelle vient remplacer l'antiracisme et les politiques construites autour de l'ethnicité. Depuis la publication en 1976 de l'étude de Naseem Khan, The Arts Britain Ignores ${ }^{5}$, sous-titrée The Arts of Ethnic Minorities in Britain, la question de «l'ethnicité » ne s'est plus départie de celle des arts. Les nombreuses polémiques que l'ouvrage avait su provoquer se transforment dans les années 1990 en une prolifération d'études sur "la diversité ethnique", tandis que dans les groupes de réflexion agissant au sein de l'Arts Council ou dans le cadre du DCMS 6 , les études se multiplient et convergent vers une affirmation du rôle positif du musée dans la société à la fin des années $1990^{7}$. L'ouvrage pionnier de François Matarasso ${ }^{8}$ publié en 1997, The Use or Ornament, dénombrait plus de 50 conséquences positives des arts sur la société, tandis que l'annexion des ressources de la loterie nationale depuis 1994 permettait un bon investissement dans le secteur artistique. En contrepartie, les secteurs de la culture devaient faire la preuve de leur efficacité à remplir un rôle aussi bien économique et social que politique. Ainsi, en donnant les pleins pouvoirs au nouveau ministère de la Culture, des Médias et du Sport, le gouvernement de Tony Blair entendait faire fructifier quatre idées essentielles: l'accès de la culture au plus grand nombre, l'excellence et l'innovation, l'éducation et les industries créatives ${ }^{9}$. Dans le champ de ses

\footnotetext{
7 Understanding the Future, Museums and the $21^{\text {st }}$ Century Londres: DCMS, 2005.

8 MATARASSO, François. The Use or Ornament.

Stroud (GB): Comedia, 1997.

9 Voir à ce sujet les documents générés par le parti travailliste annonçant le programme de Tony Blair : le New Labour Manifesto, 1997, et Ambitions for Britain, Labour's Manifesto, 2001. <www.labour-party.org.uk> (consulté en mai 2010).
} 
nouvelles compétences, les arts devraient ainsi servir les grandes lignes sociales du gouvernement, notamment en faisant de l'éducation l'outil d'intégration par excellence. Les politiques de promotion de la diversité ethnique et les politiques culturelles du gouvernement se sont fondues l'une dans l'autre. La diversité culturelle est devenue, par effet papillon, le maître mot de toutes les institutions et en particulier de l'Arts Council, qui a mis en place une stratégie de valorisation de ces termes de 1998 à 2003. Par une étrange équation, les politiques curatoriales ont visé une reconnaissance et une promotion de certains arts tels que les arts africain, caribéen, asiatique et chinois. Puis, en 2005, il appelle à la reconnaissance des identités culturelles plurielles dans Navigating Difference, Cultural Diversity and Audience Development. Cette étude liste les bénéfices économiques et sociaux qui résulteraient de la valorisation de la diversité et promulgue quelques avertissements et orientations : la diversité culturelle produit de l'innovation créative; de plus, les consommateurs issus des minorités ethniques forment des marchés inexploités; enfin, il est illégal pour toute organisation de faire de la discrimination sur des principes de race, de couleur, de nationalité ou d'origine ethnique ${ }^{10}$.

En parallèle, David Anderson, directeur des services éducatifs du Victoria and Albert Museum, signe une recherche actualisée sur les musées pour le DCMS en 1999, dans laquelle il souligne le rôle, voire la responsabilité des musées de lutter contre l'exclusion sociale. Il développe son argument: «The last two decades have seen the emergence of a 'second nation,' a substantial minority which includes a disproportionate number of young people and adults whose lives are blighted by recurrent

9 Voir à ce sujet les documents générés par le parti travailliste annonçant le programme de Tony Blair: le New Labour Manifesto, 1997, et Ambitions for Britain, Labour's Manifesto, 2001. <www.labour-party.org.uk> (consulté en mai 2010). 10 MAITLAND, Heither (dir.). Navigating Difference, Cultural Diversity and Audience Development. Londres: Arts Council England, 2005.

11 ANDERSON, A Common Wealth..., op. cit., p. 13. 12 ANDERSON, David. The Learning Age, a Renaissance for a New Britain. <http://www.lifelonglearning.co.uk/ greenpaper/>. UK Lifelong Learning/DFEE, 1998. unemployment, poor housing, poor health and drug-related crime [...] These divisions are reflected in museum audiences ${ }^{11}$. " En outre, David Anderson répond directement aux propositions de 1997 de Tony Blair dans le livre vert The Learning Age, un document en appelant à l'éducation comme nouvelle priorité ${ }^{12}$. En effet, la construction d'une nouvelle image de la Grande-Bretagne constitue l'un des fers de lance du gouvernement Blair et elle passe par une refonte de "l'imaginaire national ${ }^{13}$. De fait, les traumas des années thatchériennes et les revendications des minorités ethniques se trouveraient atténués par les termes réconciliateurs de "diversité culturelle». Les résultats directs des liens ainsi créés entre musées, intégration sociale et éducation prennent tout leur poids en devenant littéralement un impératif dans les accords de financement entre les grands musées nationaux britanniques et le DCMS ${ }^{14}$. La volonté de cibler les populations pluriethniques ou "minoritaires" dans les structures à vocation culturelle et d'agir devient un objectif national, comme en témoigne la presse des années 2000. The Independent du 6 décembre 2003 publie un article sur ces mêmes accords de financement: "No specific targets have been drawn up for numbers of visitors from ethnic minorities but agreements with the Victoria and Albert Museum and the National Gallery say that grants are linked to specific projects designed to attract a more multicultural audience ${ }^{15}$."

\section{Le Tate Britain, politiques curatoriales et politiques éducatives}

La Grande-Bretagne de la fin du XX $X^{\mathrm{e}}$ siècle cherche à redonner une cohésion à un territoire marqué par les divisions économiques,

13 ANDERSON, Benedict. L'imaginaire national, réflexions sur l'origine et l'essor du nationalisme. Paris: La Découverte,

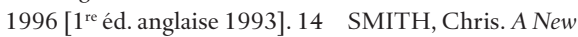
Cultural Framework. Londres: DCMS, 1998; JOWELL, Tessa. Government and the Value of Culture. Londres: DCMS, 2004.

14 SMITH, Chris. A New Cultural Framework. Londres: DCMS, 1998 ; JOWELL, Tessa. Government and the Value of Culture. Londres: DCMS, 2004.

15 BURRELL, Ian, Media and Culture Correspondent. The Independent, 6 décembre 2003. 
politiques et sociales. La volonté de la Tate Gallery of British Art de se séparer de la Tate Gallery of Modern Art émane d'un double constat: Londres n'a pas son musée d'art moderne comme ses homologues de Paris ou de New York et les artistes britanniques ont peu ou prou de visibilité dans une histoire de l'art internationale. Or, il s'agit de séparer non pas un art international d'un art national, mais un art britannique d'un art moderne. Pourquoi souligner l'absence de la Grande-Bretagne de la scène artistique moderne?

En 1993, le débat «What is British? What is Modern?" est à l'ordre du jour d'une rencontre à la Tate Gallery. Les invités ont du mal à faire des compromis. Les uns ne dissocient tout simplement pas le musée de la notion de britannicité, tel le commissaire Richard Humprey ${ }^{16}$, "The Tate is so clearly about Britishness, and a concern with modernity is peculiarly British. The question is where does twentieth-century British art fit into a Museum of modern art?", ou le directeur du Musée du design Deyan Sudjic, "Tateness is Britishness ${ }^{17}$. Tandis que Greg Hilty, fondateur de l'agence Creative Development, invalide toute lecture de l'histoire de l'art sous principe de nationalité, Nicolas Serota, directeur des différents sites des Tate Galleries, affirme la volonté d'une approche curatoriale plurielle afin de s'opposer à l'écriture et à l'illustration d'une seule grande histoire de l'art eurocentrée, exprimée par un discours homogène. Il définit l'art britannique par son insularité et son rapport aux migrations d'influences, les artistes étrangers ayant influencé les Britanniques et les artistes britanniques travaillant à l'étranger. Lors de la même discussion, le peintre royal académicien Tim Hyman conclut cependant: « We need a new relevant temple for art that the public could believe in. " Le musée d'art britannique symbolise tour à tour une église, un particularisme national, situé entre l'insularité et l'histoire

16 Qui a notamment organisé l'exposition A Picture of Britain.

17 Roundtable, Tate Archive, Tate Library Archive, non paginé, non daté. diasporique, ou bien l'expression de la théorie postmoderne de la fin des grands récits. Le Tate Britain et le Tate Modern naissent en faisant émerger conjointement la notion de «britannicité » et un discours sur la diversité culturelle.

Ce débat a des conséquences à la fois sur la collection d'œuvres des artistes artistes afro-caribéens et asiatiques britanniques et sur les politiques d'exposition de la Tate Gallery. En 1994, la Tate promeut l'Institute of International Visual Arts (Iniva) ${ }^{18}$ en accueillant le colloque Global Vision: Towards a New Internationalism. En 1995, Picturing Blackness in British Art est l'une des premières expositions à s'interroger sur le rôle et la représentation des peuples noirs britanniques à la Tate Gallery. Fidèle à son idée d'histoire de l'art plurielle, Nicolas Serota impose des acquisitions. Isaac Julien, Yinka Shonibare, Zarina Bhimji, Chris Ofili, Steve McQueen entrent ainsi dans les collections. Pendant ce temps, le contrat de financement de la Tate avec le ministère de la Culture précise:

Tate will continue to explore the historical and cultural aspects of visual art in ways that are relevant and accessible to a diverse range of visitors and encourage new audiences to engage with art. Examples include displays featuring the work of Jamaican born Ronald Moody at Tate Britain and From Tarzan to Rambo, which examines representations of race, colour and cultural stereotyping in our society, and Urban, Suburban, Rural, which contrasts artists' responses to these different environments, at Tate Modern ${ }^{19}$.

La Tate réplique en commissionnant une production de l'artiste Hew Locke lors de la British Art Week de 2004, ou en exposant Donald Rodney à l'occasion du Black History Month en octobre 2004. Les artistes d'origine afro-caribéenne sont ciblés plus

18 Le site Internet de l'Iniva explique: "Iniva was established in 1994 to address an imbalance in the representation of culturally diverse artists, curators and writers." 19 Three Year Funding Agreement 2003-2006 between the Board of Trustees of the Tate Gallery and the DCMS, Strategic Priority no. 2. Document non publié [consulté sur Tate Online en mai 2010]. 
que les artistes asiatiques ou d'autres origines. Nous pouvons émettre quelques hypothèses sur le sujet. D'une part, le mouvement artistique du Black Art et ses héritiers ont permis, depuis 1980, l'essor de questionnements autour de la blackness. Le regain d'intérêt pour Franz Fanon dans les années 1990 en est l'une des manifestations. D'autre part, les politiques d'antiracisme puis de valorisation des communautés locales ont incité de nombreux musées de ville à favoriser l'art de certaines populations, davantage représentées sur leur territoire.

Les populations noires très présentes à Londres participent de la part majoritaire des populations d'origines migratoires. Enfin, le mot Black ne désigne pas nécessairement les populations noires en Grande-Bretagne. Le terme a une valeur politique, représentative de l'Autre, celui qui n'est pas l'Anglais blanc, masculin, issu de la classe moyenne.

Lors de la création du nouveau Département des publics «Tate for All», le Tate Britain prend donc en 2003 l'engagement suivant: «Opening up our institutions to the wider community, to promote lifelong learning and social cohesion ${ }^{20}$." Felicity Allen, directrice du Département éducatif, embauche Victoria Walsh en tant que directrice du Service des publics adultes et lui demande de recruter un commissaire transculturel afin d'organiser une exposition célébrant le bicentenaire de l'abolition de l'esclavage et d'élaborer un projet de recherche qui serait présenté à l'organisme national de la recherche, l'Arts and Humanities Research Council (AHRC). Victoria Walsh parle de sa rencontre avec Mike Phillips, futur commissaire transculturel:

When Mike arrived in his late fifties, he walked: it was the last interview of the day and he put everything on the table, all the politics of Blackness, all the politics of the museum in relation to cultural diversity policy, all the fears

20 Id., p. 6.

21 Entretien avec Victoria WALSH en novembre 2008.

22 PHILLIPS, Mike et Trevor PHILLIPS. Windrush,

The Irresistible Rise of Multi-racial Britain. Londres: Harper Collins Publishers, 1998; et PHILLIPS, Mike. London Crossing: A Biography of Black Britain. Londres: Continuum International Publishing, 2001. and phobias, the internal censorship by Black people, the misrepresentation. He said everything I have never heard said by anyone ${ }^{21}$.

\section{Mike Phillips organise l'exposition Seing} Africa en 2006. Il est déjà connu pour ses livres Windrush: the Irresistible Rise of Multi-racial Britain ou London Crossing: A Biography of Black Britain ${ }^{22}$. Le partenariat Victoria WalshMike Phillips aboutit à une exposition et à un ensemble d'événements, dont le Black British Artists, une série de déjeuners-rencontres avec des artistes. Il commence à rédiger un projet en juillet-août 2005 pour l'AHRC: 50: 50 (où 50 familles seraient mis en scène à travers à 50 entretiens $^{23}$ ). En octobre 2005, Mike Phillips fait circuler une version révisée dans laquelle il redéfinit les objectifs du projet: identifier les représentations des expériences des immigrants dans les arts visuels; explorer comment les pratiques culturelles des différentes communautés y sont reflétées, si elles sont déterminées par la forme et le style des différents travaux d'artistes; identifier la manière dont la culture migratoire a nourri les différents travaux contemporains ${ }^{24}$. Quelques mois plus tard, le projet Tate Encounters voit le jour.

Le Tate Britain à cette époque met donc en corrélation deux problématiques distinctes, celle des programmes éducatifs des musées au regard de la diversité culturelle et celle des programmes curatoriaux au regard de la négation d'une histoire de l'art minoritaire, ignorée, celle des Black Artists.

\section{Tate Encounters}

Tate Encounters (2006-2010) est financé par l'Arts and Humanities Research Council, section "Diasporas, Migrations and Identity". Il réunit les collaborations du Tate Britain,

23 Des émissions télévisées, sous forme de téléréalités, déplaceraient les participants au gré de différentes circonstances dans le musée. Le cœur du projet s'attellerait à étudier les familles et les problématiques liées au statut de l'œuvre d'art. L'étude porterait sur l'accueil de la collection du Tate Britain par les participants.

24 PHILLIPS, Mike. Diasporas, Migrations and Identities: for Discussion. Document non publié, octobre 2005. 
de la London South Bank University et du Wimbledon College of Arts. Ce centre de recherche pointe les enjeux majeurs de la diversité culturelle telle qu'elle est perçue entre 1997 et 2009; en cela, il s'agit d'un projet indissociable de l'idéologie du gouvernement travailliste. En 2005, l'organe de financement de la recherche en Grande-Bretagne, l'AHRC, redéfinit ses objectifs et lance un appel à projets sur les thèmes de la diaspora, de la migration et des identités. Kim Knott, professeure d'histoire des religions à l'Université de Leeds, est nommée directrice et coordinatrice ${ }^{25}$. Elle ouvre le document de présentation de 2005 ainsi:

The challenge for an arts and humanities research programme on diasporas, migration and identities is to develop our historical and cultural knowledge about these endemic processes and how they are practised and represented, and to break new ground in how we study, theorise and model them ${ }^{26}$.

Jusque-là, les gouvernements associaient les questions migratoires à la sécurité nationale et à coup, 5,5 millions de livres sterling sont mis au service de recherches interdisciplinaires. Tate Encounters s'inscrit donc dans un panel qui rassemble des études sur des communautés identitaires, ethniques ou religieuses ${ }^{27}$. Cette mise à disposition d'un budget colossal répond à un double phénomène: d'une part, la nouvelle orientation du gouvernement et en particulier du ministre de la Culture au DCMS, David Lammy, sur les politiques de la diversité et, d'autre part, le transfert ou la responsabilité de cette union entre politique culturelle et diversité culturelle dans les instances culturelles nationales, les musées. La diversité culturelle se fait outil d'intégration culturelle par excellence et trouve son application dans les grands

25 Notons sa spécialité et sa formation de sociologue de la religion des communautés noires et sud-asiatiques en Grande-Bretagne.

26 KNOTT, Kim. Programme Specification April 2005. AHRC, Diasporas, Migration and Identity, 2005, p. 2. 27 Exemples de sujets pris en charge: représentation des femmes, «Subverting Stereotypes: Asian Women's Political Activism "; l'identité des enfants asiatiques, "Home and Away: South Asian Children's Representation and Experience of Transnationalism "; la diaspora Bengali,

musées sous la forme d'une diversification des publics. Or, cet appel à une diversification des audiences et à une prise en compte des populations minoritaires serait tout à fait louable si les instances de catégorisations ethniques ne conduisaient pas à une reproduction des discriminations et des processus d'exclusion de ces mêmes populations. La politique culturelle du gouvernement de Tony Blair a favorisé l'analyse et le développement de ces catégorisations raciales afin de générer une meilleure cohésion sociale. Par conséquent, les musées fondent leur analyse des publics sur les catégories ethniques de la Black, Asian and Minority Ethnic (BAME). Tate Encounters a pour premier objectif la remise en cause de ses formes de classification racialisée. Selon Tate Encounters, il est plus utile de partir de la reconnaissance des différences. Elles prennent en compte à la fois les vues subjectives de l'institution muséale et celles du public $^{28}$. L'équipe souhaite montrer la contradiction entre une politique de catégorisation ethnique et un effort de diversification des publics, qui se résume, selon eux, à une instrumentalisation culturelle. Les musées ont calqué leur développement de marchés sur les politiques d'intégration sociale du New Labour, il s'agit d'en interroger les conséquences.

Mike Phillips devient consultant du projet et, en octobre 2007, le directeur en charge de Tate Encounters, Andrew Dewdney, annonce les bases méthodologiques du projet, à partir de croisements de références de Bruno Latour, Irit Rogoff, Doreen Massey, Stuart Hall ou Paul Gilroy. Un document introductif en circulation dans l'équipe Tate Encounters présente les sept questions qui gouverneront la recherche. La première est: "In what ways does the Tate Collection constitute a discourse of

"Bengali Settlers in South Asia and Britain ", "Fashioning Diaspora Textile... between Britain and South Asia . À cela s'ajoutent des sujets d'anthropologie biologique ou d'histoire culturelle: "Diaspora Communities in Roman Britain"; et "Mapping Migrant Culture in Manchester 1880-2000". 28 DEWDNEY, Andrew, David DIBOSA et Victoria WALSH. «Cultural Diversity: Politics, Policy and Practices: the Case of Tate Encounters ». Document de travail non publié. 
Britishness and how does the exhibition and display programme articulate a visual imperialism in which cultural difference(s) have to be read 'against the grain"29?", et la dernière: "What factors inhibit migrant and diasporic audiences from forming meaningful and ongoing relationships with Tate, what factors enable meaningful and ongoing relationships, and can conclusions for national museums be drawn from this ${ }^{30}$ ?"

Tate Encounters souhaite relire les politiques de diversité culturelle et d'intégration sociale, les différentes conceptions de la diaspora et de l'identité nationale et interroger le rôle du Tate Britain dans ces débats. Le groupe cherche également à déterminer non seulement la définition de la britannicité du musée, mais la relation des publics à cette interprétation. Il se met en quête des non-publics, c'est-à-dire des personnes qui ne vont pas au musée. En effet, l'une des ambitions du projet est d'analyser comment faire entrer de "nouveaux publics " dans le Tate Britain. À cette fin, il forme un groupe d'analyse à partir des étudiants d'origine étrangère de la London South Bank University. Une série d'ateliers pédagogiques s'échelonne sur la durée du programme dont l'objectif est: to maximize the participation of scholars from a wide range of arts and humanities disciplines in researching, reflecting upon and discussing issues relating to diasporas, migration and identities, and their past and present impact on subjectivity and identity, culture and the imagination, place and space, emotion, politics and sociality ${ }^{31}$.

Elle prend la forme d'une rencontre avec les objets, d'une rencontre avec le domaine public et d'une rencontre dite "digitale». Aux côtés de ces séminaires pédagogiques, notons la spécificité de la recherche menée par la jeune chercheuse en études organisationnelles, Isabel Shaw, à propos des employés du Tate Britain ${ }^{32}$, notamment les collaborateurs de l'exposition The Lure of the East de 2008. Shaw renverse

29 Archives personnelles de l'équipe, documents non paginés et non publiés.

30 Id.

31 Tate Encounters, Britishness and Visual Culture.

Programme de recherche, document non publié. le terrain du projet et ouvre le panel des recherches à la réflexivité; elle pose ainsi la question de la diversité ethnique à l'équipe curatoriale et plus globalement aux membres du Tate Britain. L'anthropologue visuelle Sarah Thomas élabore le pendant de cette étude lorsqu'elle réalise des films documentaires ethnographiques sur les participants-étudiants et leur cadre familial. Les deux travaux mettent ainsi face à face les employés du musée et les publics ou les non-publics.

La spécificité du projet tient donc également à l'hétérogénéité des travaux engagés. Loin de former une constellation de chercheurs unis sur une même question, l'équipe est divisée quant aux compétences, mais également quant aux méthodes, pratiques et types d'objectifs. David Dibosa concentre son attention sur le rôle du spectateur, l'épistémologie de la migration grâce à des interviews filmées des étudiants. Sarah Thomas, avec un groupe d'étudiants, produit à la fois des vidéos et des textes d'études anthropologiques. Mike Phillips s'occupe de la représentation historique des peuples noirs, des politiques culturelles et des politiques curatoriales, sur un mode principalement autobiographique. Victoria Walsh propose une mise en contexte et une étude des relations entre le Tate Britain, le Service éducatif et les politiques culturelles de la diversité, tandis qu'Isabel Shaw développe une étude organisationnelle des employés du Tate Britain. Andrew Dewdney dirige et développe l'assise méthodologique du groupe et encadre les groupes d'étudiants afin de produire une étude quantitative des publics et des non-publics du musée, tout en poursuivant des études spécifiques sur les nouvelles technologies en science de l'éducation dans ses ateliers. La pluralité des représentations du projet et ses états éphémères engendrent une difficulté de ses publics à saisir les enjeux. En effet, les groupes d'étudiants participants manifestent leur difficulté à décrire les objectifs

32 ALVESSON, Mats et Kaj SKÖLDBERG. Reflexive Methodology, New Vistas for Qualitative Research. Londres: Thousand Oaks / New Delhi: Sage Publication, 2000. 
du projet, ou encore le rôle des membres de l'équipe. Alors que Jacqueline Ryan, étudiante, explique que le projet a pour but de proposer un reflet culturel de la société qui permettrait au musée d'attirer davantage de publics issus des minorités ethniques ${ }^{33}$, Patrick Trubidy, un autre participant au projet, déclare: «la britannicité ne fait plus partie de ce projet depuis longtemps. Je ne sais pas pourquoi il a perdu en route 'sa britannicité', mais il a dérivé pour interroger le musée lui-même, 'quel est le rôle du musée? ${ }^{34}$. Chacun des participants au programme sera amené à écrire un essai ou à réaliser une vidéo qui rende compte de son sentiment à propos de Tate Encounters, une manière d'appréhender la compréhension de la britannicité, ses enjeux et les rôles du musée selon d'autres publics. La plupart d'entre eux suggéreront que le musée est davantage un lieu de contrôle et de régulation qui opère une restriction plutôt qu'une expression des identités ${ }^{35}$.

J'ai découvert le programme Tate Encounters torat portant sur la britannicité dans les arts contemporains. Je cherchais les traces et les connexions entre les programmes des partis politiques menés successivement par les gouvernements Thatcher et Blair, et l'intérêt accru pour la question de la Britishness dans les mondes de l'art britanniques (Howard Becker). En effet, la notion d'appartenance à une identité collective semblait dominer les scènes politiques, culturelles et artistiques depuis la fin des années 1970. Les années de gouvernance conservatrice avaient divisé le corps social, engendré une opposition entre des programmes pédagogiques communautaires et l'éducation nationale, entre les artistes et le pouvoir en place. Tate Encounters est devenu l'un

33 Entretien avec Jacqueline RYAN en novembre 2008: "We often criticised the Tate Britain, through it you get a grasp on what the projects goals and ideas are. To make a reflexion on society and in doing that: hopefully it is going to be more accessible and attractive to a different audience especially to people from ethnic minorities background."

34 "SO: Do you think the project is still about Britishness? PT: No it left Britishness a long time ago. I don't know why it lost its Britishness but it drifted back about the Tate, what is the Tate's role... " À qui l'étude s'adresse-t-elle? Il faut noter que, chemin faisant, l'ambition du projet a surtout dépassé

des terreaux de ma réflexion. L'équipe m’a accueillie et a accepté que je passe quelques mois d'observation en leur compagnie. Je troquais donc la méthodologie des cultures visuelles et de l'histoire de l'art au profit d'une anthropologie participative. J'ai pu ainsi me glisser dans les réunions, dans les ateliers avec les participants, observer les méthodologies, les comportements, l'évolution du travail, tout en menant des entretiens avec chacun des membres de l'équipe et avec certains étudiants. Ma prise de notes, mes recherches et mes participations se sont déroulées de septembre 2008 à mars 2009, jusqu'à la première grande présentation et expérimentation publique du laboratoire de Tate Encounters.

Le groupe de recherche n'avait pas encore livré ses résultats définitifs à cette date. Toutefois, des éléments d'analyse étaient déjà présents dans l'exposition Tate Encounters: Research in Process présentée du 23 février au 20 mars 2009 au Tate Britain. Elle était l'occasion de montrer les travaux des "chercheurs associés" et de proposer quatre semaines de colloque sur les thèmes de recherche du groupe ${ }^{36}$. Le document End Games (non publié) circulait à ce moment-là dans l'équipe. Andrew Dewdney y éclaircissait la méthode de travail avec les participants. Les "chercheurs associés " y étaient définis comme partie prenante d'une triangulation "projet-participant-investigateur ", à travers «la création d'une compréhension critique par un dialogue réflexif ». Il faut sans doute voir là l'influence de Mats Alvesson et Kaj Sköldberg ${ }^{37}$ et de leurs travaux sur la méthode réflexive dans les recherches qualitatives. Les participants au projet ou "chercheurs associés » étaient donc placés au cœur du dispositif de création de connaissance. Ils formaient également un nouveau public. Ils incarnaient

les attentes initiales et que la surcharge de données et de matériel de réflexion ont fait du projet une sorte de machine autonome dont chacun des membres est impatient d'étudier les différents angles et le fonctionnement.

35 DEWDNEY, DIBOSA et WALSH. "Cultural Diversity: Politics, Policy and Practices..." op. cit., p. 12.

36 <http://process.tateencounters.org/>

(consulté en mai 2009).

37 ALVESSON et SKÖLDBERG, Reflexive Methodology, New Vistas..., op. cit. 
la nouvelle identité plurielle du "Britannique". "Non-publics", ils s'écartaient d'un modèle de public: le visiteur classique, Anglais, blanc, de classe moyenne ou de la haute bourgeoisie. Les étudiants incarnaient donc la voix des exclus, qui ne se reconnaissent pas dans la britannicité écrite, exposée par le Tate Britain. Si ce "subalterne parle», selon l'expression de Gayatri C. Spivak, il est un autodidacte compétent, créatif, pertinent, à opposer au visiteur passif, cet obligé d'une histoire de l'art dans laquelle il ne se reconnaît pas, mais qui appartient à l'histoire de la nation. Il devient, à terme, le producteur d'un autre récit à la marge de l'histoire des représentations et nourrit une nouvelle muséologie. Celle-ci s'oppose aux méthodologies d'histoire de l'art des départements curatoriaux au profit d'une méthodologie issue des connaissances du spectateur lui-même. Non seulement le groupe interrogeait la manière dont les définitions de la britannicité sont produites et reproduites par l'institution, mais il développait un contrepouvoir ou une réponse critique.

De fait, l'exposition Tate Encounters: Research in Process permet de lire les différents niveaux de recherche traités par l'équipe: la généalogie des services éducatifs, le rôle du numérique dans les musées et la représentation de la migration par le musée et à sa réception, en particulier par les étudiants, invités à produire une œuvre ou un film ethnographique. La quatrième semaine d'exposition de réflexion portait sur les relations entre art et politique. Tate Encounters exposait non pas les œuvres, mais les questions, les débats, les réceptions des programmes du Tate Britain. Le groupe exposait les enjeux de la britannicité définie par le musée, il se mettait en scène, il installait son bureau au centre d'un espace dédié à une autre fonction, il fonctionnait comme une critique institutionnelle en acte. Le lieu d'exposition, en tant qu'espace de recherche, déplaçait la fonction critique du projet, son ambition, sa complexité, pour l'installer dans l'antre de

38 PRATT, Mary Louise. "Arts of the Contact Zone" [1991], version électronique tirée de BARTHOLOMAE, David et Anthony PETROKSKY (dir.). Ways of Reading. New York, Bedford: St. Martin's, 1999 [5 $5^{\mathrm{e}}$ ed.]. <http://web.nwe. ufl.edu/ -stripp/2504/pratt.html> (consulté en mai 2010). la machine des Tate Galleries au cour des rapports de pouvoirs entre commissaires (historiens de l'art) et services éducatifs, entre machines de reproduction des savoirs et volonté de réformer les méthodes de diffusion et d'apprentissage. Le contenu, les films, les débats comptaient tout autant que le processus et la décision de faire cette exposition. Tate Encounters était littéralement une "zone contact" au sens de MarieLouise Pratt ${ }^{38}$, entre les publics, la recherche, les musées, un lieu "réflexif" sur les pratiques muséologiques, les enjeux des services éducatifs et des services de gestion des publics.

\section{Le tournant éducatif des musées et questionnement des programmes curatoriaux}

\section{La particularité d'un projet tel que Tate}

Encounters est de se situer à la lisière de plusieurs services du musée et, de fait, de questionner à nouveau le rôle d'un musée national, historiquement associé à l'éducation. Comme David Anderson le souligne ${ }^{39}$, l'éducation dans les musées était déjà une préoccupation du XVIII siècle. René Huyge, conservateur du Louvre note d'ailleurs la naissance conjointe de l'encyclopédie et du musée. Kenneth Hudson reprendra l'explication en ces termes: "Both were an expression of the eighteenthcentury spirit of enlightenment which produced an enthusiasm for equality of opportunity of learning... The theory behind these movements was a simple one, that collections which had hither to been reserved for the pleasure and instruction of few people should be made accessible for everybod $y^{40}$." Cependant, les pratiques d'enseignement et surtout l'idée d'éducation auraient récemment changé au profit d'une vision plus individualisée, plaçant l'expérience au centre de l'apprentissage. George E. Hein situe les origines de ce déplacement de la définition de l'éducation comme participation du spectateur ou de «l'apprenti » dans son environnement, dans la philosophie de John Dewey

39 ANDERSON, David. A Common Wealth..., op. cit. 40 HUDSON, Kenneth. A Social History of the Museum, What the Visitor Thought. Londres: McMillan, 1975, p. 6. 
et la psychologie de Jean Piaget, qui reconnaissent l'importance de l'apprentissage par l'expérience. Une opposition se serait donc créée entre

l'apprentissage formel de l'école, rébarbatif et soumis à un programme, et celui du musée, informel, libre ${ }^{41}$. Eilean Hooper-Greenhill analyse plus globalement le changement du rôle des musées et définit "un post-musée " faisant suite au "postmoderne». Ce postmusée se caractérise par un nouveau rapport aux publics déterminé par les relations entre culture, communication, apprentissage et identité et également par sa capacité à promouvoir une société plus juste et plus égalitaire ${ }^{42}$.

Cet intérêt croissant pour une discipline considérée comme mineure dans beaucoup de musées européens est également marqué par «la nouvelle muséologie» initiée par Peter Vergo en 1989. Proposant une histoire de la muséologie dans laquelle les fonctions de lieu d'apprentissage et lieu d'exposition cheminent à parts égales, Vergo invite des historiens ou des assistants de conservateur, des spécialistes de cultural studies ou d'arts visuels, à placer au centre du débat et à examiner le rôle du musée en s'affranchissant de l'obsession de méthode de «l'ancienne muséologie». On peut noter également le développement des départements de muséologie et en particulier celui de Leicester ou celui de l'Open University en Grande-Bretagne, qui se préoccupent largement des fonctions du musée et des enjeux de la diversité culturelle ${ }^{43}$. Max Ross, dans un article paru dans Museum and Society ${ }^{44}$, définit la nouvelle muséologie comme la réflexivité institutionnelle émergeant dans les années 1970, transformant le musée de lieu d'exclusion

41 HEIN, George E. Learning in the Museum. New York, Londres: Routledge, 1998, p. 9: «In Britain, the introduction of a national curriculum in 1989 gave rise to publications (Moffat 1996, School Curriculum and Assessment Authority 1995, Yorath 1995) that discussed the use of museums to support a statemandated education goal."

42 HOOPER-GREENHILL, Eilean. Museums and Education. Purpose, Pedagogy, Performance. New York, Londres: Routledge, 2007, p. 1.

43 À ce sujet, se référer aux travaux de Richard Sandell de l'Université de Leicester ou de Tony Bennett. SANDELL, Richard. Museum, Society Inequality. Londres, New York: Routledge, 2002; BENNETT, Tony. «Civic Laboratories: et de division sociale en un lieu à haute responsabilité d'intégration sociale. Dans un autre numéro de cette revue créée par le Département de muséologie de l'Université de Leicester, Colin Trodd tente d'analyser comment l'histoire de l'art perçoit la muséologie. Il revient en particulier sur l'une des tendances des analyses concernant la fonction critique du musée dans les années 1990 à 2000.
Art museums have been identified as places where the rituals of citizenship are enacted, where disciplinary technologies are active, where governmental forces are located and where political legitimations are made [...] the postmodernist, post-structuralist and neo-marxians researches of Douglas Crimp, Carol Duncan and Tony Bennett discover a panoply of forces, powers and technologies for the organisation and control of experience and meaning [...] Art, it was claimed, could be re-tooled through Literary and Critical Theory, Cultural Studies, Anthropology and Sociology; and the critical evaluation of the art museum was an important component of this new 'heteroglossic' formation ${ }^{45}$.

Selon Trodd, ces théories oublient que le musée expose différentes formes de pensées, en assignant au "voir" " quelque chose de discipliné par un mécanisme extérieur de production de savoir ${ }^{46}$ qui rationalise le musée en tant que machine à programmes idéologiques. Ainsi, en Grande-Bretagne et en particulier à l'Open University, les départements d'histoire de l'art s'accordent à associer le musée à une forme de contrôle social, voire d'impérialisme (Tony Bennett ${ }^{47}$ ). Dans une verve similaire, Felicity Allen, responsable du Département interprétation et apprentissage au Tate Britain,

Museums, Cultural Objecthood, and the Governance of the Social ". In. CRESC. Open University, Working Paper Series, $\mathrm{n}^{\circ} 2$, mai 2005.

44 ROSS, Max. «Interpreting the New Museology».

Museum and Society, vol. 2, $\mathrm{n}^{\circ}$ 2, 2004.

45 TRODD, Colin. «The Discipline of Pleasure; or, How

Art History Looks at the Art Museum ». Museum and Society, vol. $1 \mathrm{n}^{\circ} 1,2003$, p. 18.

46 Id.

47 BENNETT, Tony. "The Exhibitionary Complex". In. BOSWELL, David et Jessica EVANS (dir.). Representing the Nation: a Reader - Histories, Heritage and Museums.

Londres: Routledge, p. 332-361. 
explique comment le développement des services éducatifs à partir du milieu des années 1980 est passé d'un statut d'expérimentation à celui de pilier de l'économie créative, autrement dit, pilier des études sur les publics, tout en s'inspirant des mouvements de certaines gauches.

I argue that gallery education, as it developed since the mid-1970s, has been both a distinct and overlapping artistic strategy which is integrally connected to radical art practices linked to values aired and explored in the liberation movements of the 1960s and 70s, and particularly the women's movement. It is an individual strategy among many (including, for instance, small-scale exhibitions, small press and small magazine publishing, alternative libraries and archives), to shift art from monolithic and narcissistic position into a dialogic, open, and pluralist set of tendencies that renegotiate issues of representation, institutional critique and inter-disciplinarity ${ }^{48}$.

Le programme d'exposition de Tate Encounters réalisé en février et mars 2009 est sans doute le cas le plus abouti de recherche rendue publique sur la "britannicité et les arts visuels", autrement dit sur les enjeux et les problèmes de la "diversité culturelle» en liant une histoire de l'éducation muséale et une pratique éducative nourrie par un parti pris anthropologique au sein d'une exposition-témoin, sorte de laboratoire en démonstration dans l'une des salles du Tate Britain. L'événement se situe dans cette généalogie de formes d'enseignement et de transmission du savoir en marge de l'éducation nationale. Il n'est donc pas loin de l'action culturelle et associe des objets hétérogènes mais reliés, comme une analyse des subjectivités des jeunes étudiants porteurs d'un patrimoine migratoire, à un état des lieux des services éducatifs, à la création d'un savoir anthropologique sur les publics ou les nonpublics du Tate Britain.

Au moment même où ce laboratoire d'idées, installé au cœur des différentes institutions de l'art (les beaux-arts, l'université, le musée national), se met en action, les politiques curatoriales du Tate Britain interrogent la nécessité de représenter et de rendre visibles des artistes issus des vagues d'immigration du Commonwealth, les Black Artists, jusque-là ignorés par cette institution. Mike Phillips laisse son poste à Paul Goodwin, jeune professeur au Goldsmith's College. Goodwin est spécialiste des relations entre les cultures noires et la ville, l'urbanisme. Il s'intéresse à une théorie critique liant les espaces à une politique des Black Urbanisms et il dirige alors des séminaires sur le sujet au City Hall de Londres. Il devient le nouveau commissaire des transcultures. Il s'applique à interroger l'histoire, la "narration" produite par les commissaires, par le biais d'une série de conférences et d'expositions. C'est ainsi qu'il organise en 2009 une réflexion sur les expositions internationales qui relient l'art contemporain et la diaspora africaine; il pense les enjeux des « rencontres urbaines ", il dirige une conférence sur la notion de frontière, tout en engageant un dialogue sur la collection du Tate Britain et une collaboration avec les artistes. Il produit une exposition sur le Black Audio Film Collective, puissant collectif artistique de films et de vidéos créé en 1982 par sept réalisateurs, dont John Akhomfrah (Handsworth Songs, 1986) et Reece Auguiste (Twilight City, 1989). Tout récemment, Goodwin propose de reprendre l'accrochage de l'exposition The Thin Black Line avec Lubaina Himid, organisée par celleci à l'Institute of Contemporary Art en 1985, exposition qui s'était révélée comme l'un des marqueurs de l'histoire des Black Women Artists de Grande-Bretagne.

Les années 2006-2011 constituent, de fait, une période très spécifique de l'histoire du Tate Britain où le débat sur la diversité culturelle fait émerger les objets cachés, les tabous, les non-dits et les met au centre des discussions, en demande de redéfinition. En 2009, le musée fait appel au commissaire d'exposition français Nicolas Bourriaud et à son «esthétique relationnelle " pour organiser la triennale d'art contemporain. Habituellement dédié 
aux artistes britanniques, cet événement vise cette fois à redéfinir le rapport des artistes à la mondialisation en tablant sur une " altermodernité ". L'altermodernisme caractérise une sortie d'un modèle moderne au profit d'un modèle multiculturaliste, adapté à un artiste nomade, réceptacle d'une identité stratifiée, créolisée, construite au gré de ses pérégrinations, dans un univers hypertextuel, hypermédiatique. Bourriaud semble vouloir composer ce nouveau public créatif des musées, à partir d'une nouvelle figure de l'artiste, lui aussi soumis au principe de la diversité culturelle. Ici, il s'agit de défaire les représentations modernes de l'artiste, de désenclaver la muséologie de ses habitudes d'accrochage, en proposant une lecture concertée, collaborative, des travaux avec d'autres chercheurs comme Okwui Enwezor, T.J. Demos, Carsten Höller. Cette expérience curatoriale partagée n'est pas loin de certaines orientations des expositions internationales alternatives dont le premier opus serait la biennale de Cuba (à partir de 1984), contestataire, unificatrice des pays émergents du deuxième et du troisième mondes, face à une domination européano-américaine. Gerardo Mosquera, commissaire d'exposition, critique d'art et historien de l'art vivant à la Havane souhaitait écrire un contre-récit de la modernité, par la présentation d'artistes internationaux sous le principe de l'éradication des hiérarchies et la promotion d'un esprit d'égalité. L'exposition Altermodern ne reprend pas l'égalitarisme des années 1980, mais transpose des techniques curatoriales dans une époque qui ne peut plus faire l'impasse de la mondialisation. La triennale symbolise le questionnement de l'équipe curatoriale du Tate Britain à propos d'une négociation éventuelle entre l'art britannique, sa britannicité et l'art contemporain mondialisé. Si les acquisitions d'œuvres d'artistes britanniques du Black Art sont encore ponctuelles, les expositions temporaires doivent interroger les possibles relectures, décentrages, renversements de l'hégémonie d'une histoire de l'art occidental moderne. En 2011 néanmoins, l'heure est aux bilans. Le programme Tate Encounters s'achève, Andrew Dewdney, Victoria Walsh et David Dibosa s'attèlent à la rédaction d'un ouvrage, tandis que le contrat de Paul Goodwin n'a pas été renouvelé. La fin du mandat du Tony Blair a provoqué un changement de direction dans les politiques culturelles. Tate Encounters a accumulé au cours des cinq dernières années une énorme quantité de matériel, un volume qui dépasse les limites d'un programme de recherche et un foyer multidirectionnel d'objets, de documents, d'entretiens, de discussions et de productions.

\section{Premiers résultats et conclusion}

Depuis juin 2010, l'équipe rend compte de recherches dans des domaines spécifiques: les pratiques curatoriales et l'histoire de la britannicité, la diversité culturelle et le rôle du musée et les méthodologies des services éducatifs des musées. Sont ainsi générés 300 questionnaires, 200 dissertations sur le Tate Modern et le Tate Britain, 12 ateliers de travail différents, autant de recherches individualisés avec des participants, des films ethnographiques et 38 entretiens avec les employés du Tate Britain. Les premières conclusions font état de discours raciaux dans les pratiques de la diversité culturelle et de l'absence d'une réflexion aboutie sur le sujet dans les services de gestion des publics ${ }^{49}$. Il est à noter que les définitions du public incombent tout à la fois au Service marketing, au Service curatorial et au Service éducatif. Cette absence d'analyse poussée des publics conduit à la reproduction des écarts, des préjugés et des divisions dans les différentes taxonomies des publics, au mépris d'une conception fluide, construite et nuancée de l'identité des non-publics, comme le prouvent les essais et les productions des participants au programme Tate Encounters.

49 [E]dition 6, "Tate Encounters: Selected sections from final project report submitted to the Arts and Humanities Research Council (juin 2010)". <http://www.tate.org. $\mathrm{uk} /$ research/tateresearch/majorprojects/tate-encounters/ edition-6/papers.shtm> (consulté en septembre 2011). 
L'expérience de la migration, l'élaboration d'un patrimoine culturel par chacun des sujets associés à l'enquête ont montré l'inadéquation des attentes et surtout des représentations entre les publics ou les non-publics identifiés par le musée et ses visiteurs. En réalité, les politiques de "représentations du sujet » ne sont plus à même de traduire la manière dont les expériences culturelles migratoires ont formé les nouvelles générations de Britanniques:

While the politics of identity and representation were actively pursued as a part of the project of multiculturalism in the 1980s and 1990s, freed and informed by the debates of postcolonialism of interest to a generation defined by post-war migration from the Colonies, third generation migrational peoples resist concepts of fixed identity, and demonstrate a significant shift towards the concept of fluid subjectivity and cultural hybridity ${ }^{50}$.

Les recherches ethnographiques, les ateliers menés avec les participants au projet Tate Encounters sont parvenus à l'appréhension d'un sujet "transculturel", habité par des savoirs transmédiatiques et transvisuels. Ainsi, les différents programmes éducatifs, autant que les accrochages des collections et l'histoire identitaire et culturelle portée par le Tate Britain, se construisent et alimentent et reproduisent «une représentation» erronée des publics.

Par ailleurs, les analyses des données empiriques ont mené à une mise en cause du modèle curatorial moderne du "cube blanc", face au déploiement des nouveaux moyens d'accès à la connaissance, garantis par les nouvelles technologies. Ici, l'équipe a cherché à développer les conditions de négociation, d'apprentissage, de participation entre un public et un objet visuel. Tate Online pourrait ainsi représenter un champ d'exploitation intersubjectif, interactif, des relations des individus à l'histoire de l'art. Un nouvel assemblage théorique semble possible, venant concurrencer les principes plus figés des pratiques curatoriales issues du XIX ${ }^{\mathrm{e}}$ siècle.

50 DEWDNEY, Andrew, David DIBOSA et Victoria WALSH. Tate Encounters, Britishness and Visual Culture 2007 2010. Londres: AHRC, 2011, p. 24.
L'apport du projet Tate Encounters est également à situer du côté de la méthodologie. Si l'interdisciplinarité est le mot clé des projets universitaires depuis une vingtaine d'années, peu de laboratoires ont prouvé l'efficacité concrète de cette entreprise. Tate Encounters n'a cessé de remettre en cause l'interdisciplinarité, non seulement en tant qu'outil d'analyse, mais comme appareil de décentrement des certitudes, des conventions, des habitudes de la muséologie et des cultures visuelles. Tate Encounters est un laboratoire d'expérimentations, d'analyses, d'échanges des outils de pensée, des méthodes de travail, des objets de réflexion et il produit une généalogie des disciplines en présence. Durant les mois d'observation en compagnie de l'équipe, j'ai pu remarquer que, au-delà de la densité des projets, de l'engagement des membres et des strates de matériaux produits, les réunions revenaient incessamment sur la difficulté à donner du sens et à donner une forme à l'interdisciplinarité ou plutôt à la transdisciplinarité. Si le projet avait pris ses sources dans l'affinité entre l'histoire de l'art, l'anthropologie et la sociologie, sa mise en pratique a opéré un déplacement théorique vers d'autres territoires. La muséologie, la culture visuelle et les sciences sociales, les théories de la cybernétique, l'anthropologie visuelle, les études organisationnelles ont trouvé les accroches, les points de liaison qui permettent l'entrelacement de leurs outils, autrement dit la transdisciplinarité. Les assemblages de matières théoriques et leurs usages communs n'ont pas été créés dans la facilité, mais dans la tension, dans la négociation, dans un effort constant de chacun des membres de l'équipe. En juin 2010, Andrew Dewdney, David Dibosa et Victoria Walsh rendent compte de leurs travaux sur trois sujets, chacun regroupant des champs disciplinaires variés dans le dernier opus de la revue en ligne [E] dition $^{51}$. Ainsi, le premier domaine concerne les politiques culturelles, le deuxième unit les arts du management et les industries culturelles, le troisième assemble la muséologie, les études culturelles et la culture populaire.

51 [E]dition 6, "Tate Encounters: Selected sections...", op.cit. 
Le croisement de ces domaines et leurs expérimentations par et auprès des participants fondent également l'un des enjeux forts de la méthodologie de Tate Encounters, celui d'une théorie fondée sur la pratique et sur la réflexivité, autrement dit l'analyse des enjeux des méthodes utilisées pour les enquêtes, les ateliers et les travaux, un retour critique constant sur les matériaux et les informations générés par la recherche. La collaboration avec les participants, les "chercheurs associés", ont abouti à la conclusion que les musées sont des lieux de rencontres, des zones de contacts qui génèrent du sens ou de la valeur (value), qu'il convient de soutenir et de rendre visibles dans la pratique de la muséologie. Ainsi, l'analyse des publics a ici été construite à partir de l'expérience des spectateurs en leur proposant de se situer et de produire une réflexion sur les programmes et les collections du musée. Tate Encounters qualifie sa méthodologie de "muséologie postcritique", une métadiscipline

[w] hich has developed from the contrast between the conditions of the production of project's own embedded, transdisciplinary, action-orientated collaborative approach and that of other traditional research approaches in which the formal division between the academic research community and the subject of research is reproduced ${ }^{52}$.

Tate Encounters livre enfin une analyse extrêmement fine des relations entre politique et culture en jeu dans la promotion de la diversité culturelle du Tate Britain de 1997 à 2010. Ainsi, le Tate Britain a su être à la fois initiateur, maison d'accueil d'une réflexion pionnière, contestateur de l'analyse de la gestion du sujet et du public, tout en déployant des formes de résistance à la réflexivité intrinsèque à ce projet et à ses conclusions. L'une de ces stratégies de résistance est l'évitement, le déni, et s'illustre par exemple par un silence à propos du manque d'artistes "noirs britanniques " dans la collection. La promotion de la diversité culturelle a été, en ce sens, la couverture politique, sociale et historique du malaise 
By Sophie Orlando, translated by Philippe Lagrange

The new role of museums and cultural diversity: Case study
of Tate Encounters: Britishness and Visual Culture, Tate, Britain

From 1997 to 2010, Tony Blair's government launched a policy of "cultural diversity" in order to respond to the new image of Great Britain; multiculturalist, pluralist, inclusive. The creation of the Tate Modern museum in the 2000's generated the division between international contemporary art and art historically the bearer of "Britishness". How is national art defined and how is it possible to integrate artists from different origins when building a visual homogenous narrative? The hanging of collections, educational policies, and the diffusion of the museal knowledge deliver a story of British history. The strong leanings of Blair's government lead to the questioning of curatorial, educational and marketing policies and their association to multiculturalist identity policies. The Tate Britain will have to negotiate with the governmental interpretation of the contemporary Brit, heir to the colonial empire, stuck between a national Victorian history and the aftermath of decolonization. In that respect, the museum will display its definitions of the public according to ethnicity classification (BAME), thus recreating a concept of identity that is racialized, fixed and predetermined.

The Tate Encounters program was born from the necessity to question the application of policies on cultural diversity in the Tate Britain, and the influence of these policies the public. Andrew Dewdney, David Dibosa et Victoria Walsh will be coordinating a research program, a laboratory for applied theory, thanks to financing provided by the Arts and Humanities Researches Council, linking three institutions addressing the philosophy and practices of arts, the school of art (Chelsea College), the university (London South Bank University) and the museum (Tate Britain). The interdisciplinarity of art history, sociology, and anthropology will serve as a common primary structure to facilitate the analysis of the public, but primarily the "non-public". The financing of national museums is increasingly characterised as being autonomous; the institutions must widen their audiences and lead studies and campaigns targeting specific communities. The specific government demand for the diversification of the public triggered an increased interest for migratory populations or those with a diasporic history. Tate Encounters tackles the relationship between the public, the museum and the narration of a national history entitled "Tate Encounters, Britishness and Visual Culture".

What are the stakes of this research program and what are the challenges resulting from an experiment lasting from 2006 to 2011? How to analyze this program while taking in account the policies for cultural diversity promoted by the Tate Britain and what are the primary conclusions drawn from the work done at the Tate Encounters?

This article explores the links between the birth of a policy for ethnic diversity as well as cultural diversity within the government at the time of the division of the Tate Gallery into a building both dedicated to 
internationalism and to the modern interpretation of the concept of national identity. Also, it analyzes the circumstances from which the Tate Encounters project has emerged and observes its theoretical evolution by focusing on the exhibit Tate Encounters: Research in Progress, public expression and methodological, critical and theoretical confrontation of the main concerns of the research team and its participants. Finally, the paper focuses on certain aspects of the program and discusses some results, particularly the methodology. 